\title{
El conocimiento \\ según las ciencias cognitivas
}

\author{
Knowledge \\ according to cognitive sciences
}

\author{
PASCUAL F. MARTÍNEZ-FREIRE \\ Universidad de Málaga
}

\begin{abstract}
RESUMEN
Después de una breve caracterización de las ciencias cognitivas, se destaca la existencia de dos enfoques erróneos en filosofía del conocimiento (uno erudito-histórico y otro meramente especulativo) apostando por el enfoque propio de las ciencias cognitivas, donde el sujeto de cognición es procesador de información (siguiendo la idea de Alan Turing) y tanto puede ser un humano como una máquina o un animal. En segundo lugar se caracteriza el sujeto cognitivo como un sistema físico de símbolos (SFS), de acuerdo con la tesis de Allen Newell y Herbert Simon, comentando y ejemplificando tal tesis. Y finalmente se distingue entre diversos grados de cognición en sentido amplio, tanto en animales como en máquinas, así como se destaca la cognición en sentido propio o cognición inteligente, que comprende las operaciones de concebir, juzgar y razonar.
\end{abstract}

\section{PALABRAS CLAVES}

CONOCIMIENTO, COGNICIÓN, TURING, NEWELL, SIMON, COGNICIÓN PROPIA

\begin{abstract}
After a brief description of cognitive sciences, the existence of two mistaken approaches in philosophy of knowledge is emphasized (the one is the historical-scholar and the other is the merely speculative) betting on the approach proper of cognitive sciences, where the subject of cognition is an information processor (following Alan Turing's idea) and it can be not only a human but also a machine or an animal. Secondly the cognitive subject is characterized as a physical symbol system (PSS), according to the thesis by Allen Newell and Herbert Simon, and such thesis is commented and illustrated. And finally diverse degrees of cognition in general
\end{abstract}

(C) Contrastes. Revista Internacional de Filosofia, vol. XXIII-N² (2018), pp. 115-123. ISSN: 1136-4076

Departamento de Filosofía, Universidad de Málaga, Facultad de Filosofía y Letras Campus de Teatinos, E-29071 Málaga (España) 
sense, in animals and in machines, are distinguished, but also it is highlighted the proper or intelligent cognition, comprising the operations of conceiving, judging and reasoning.

KEY WORDS

KNOWLEDGE, COGNITION, TURING, NEWELL, SIMON, PROPER COGNITION

\section{EL CONTEXTO: EL CAMPO INTERDISCIPLINAR DE LAS CIENCIAS COGNITIVAS.}

INTERESA RECORDAR, aunque brevemente, algunos datos acerca del surgimiento y caracteres de las ciencias cognitivas.

La propia denominación de «ciencias cognitivas» data del año de 1975. En efecto, en ese año tiene lugar la publicación de dos importantes libros donde se hace referencia explícita al término «ciencia cognitiva». Se trata, por un lado, de la obra editada por Daniel G. Bobrow (1935-2017) y Allan Collins titulada Representation and Understanding. Studies in Cognitive Science, y asimismo, por otro lado, de la obra editada por Donald A. Norman y David E. Rumelhart (1942-2011) titulada Explorations in Cognition. Al final de este último libro se dice: «Los esfuerzos concertados de un número de personas procedentes de las disciplinas relacionadas de lingüística, inteligencia artificial y psicología puede estar creando un nuevo campo: ciencia cognitiva».

Por otra parte en 1976 se inició la publicación de la revista Cognitive Science, que aún se sigue publicando, mientras que en 1979 se constituyó la Cognitive Science Society, que desde entonces celebra un congreso anual.

En cuanto a las ciencias integrantes de este campo interdisciplinar, en la cubierta de la revista Cognitive Science aparece un laberinto octogonal con los nombres en su alrededor de siete disciplinas: lingüística, neurociencia, filosofía, psicología, antropología, inteligencia artificial y educación (pedagogía). Ahora bien, y tal como he señalado en La importancia del conocimiento (2007, pp. 32-33), debemos distinguir entre aquellas disciplinas que constituyen el núcleo de este campo interdisciplinar y aquellas otras disciplinas implicadas de manera instrumental o bien solo en parte. A mi entender las dos ciencias cognitivas básicas son la psicología de orientación cognitiva y la ciencia de la inteligencia artificial. La ciencia de la inteligencia artificial fue lanzada al gran público en el verano de 1956, en el Dartmouth College de la ciudad de Hanover (New Hampshire), por un grupo de diez matemáticos y lógicos, entre los que estaban John McCarthy (1927-2011), Marvin Minsky (1927-2016), Allen Newell (19271992) y Herbert Simon (1916-2001). Tal disciplina nacía como una rama de la informática que estudiaba la teoría, el diseño y la construcción de máquinas inteligentes. A su vez, la psicología de orientación cognitiva puede considerarse constituida en 1960, cuando los psicólogos George A. Miller (1920-2012) y Jerome Bruner (1915-2016) fundan el Harvard Center for Cognitive Studies 
en la Universidad de Harvard. La psicología cognitiva recupera el mentalismo (o defensa de la existencia de una vida mental interna) frente al conductismo, reinante desde 1913, aunque por supuesto no renuncia al análisis de la conducta.

\section{Del sujeto De CONOcimiento AL SUjeto COGNitivo.}

En mi trabajo «El impacto de las ciencias cognitivas en la filosofía del conocimiento» (1995, pp. 52-53) me he referido a los enfoques erróneos en la filosofía del conocimiento. Por supuesto la teoría o filosofía del conocimiento es una disciplina filosófica que se remonta a los comienzos de la actividad intelectual en la Grecia clásica. Pensemos, por ejemplo, en el Menón de Platón (428-347). No tiene nada de sorprendente que así haya ocurrido puesto que las cuestiones acerca de la naturaleza, fuentes y alcance (límites) del conocimiento preocupan al ser humano justamente en cuanto sus conocimientos pretenden ser objetivos y fundamentados.

Sin embargo, el estudio de los problemas del conocimiento, tal como deben abordarse en los tiempos actuales, debe evitar dos enfoques que podemos considerar erróneos, a saber, el enfoque erudito-histórico y el enfoque meramente especulativo. Por una parte, poco avance real cabe esperar si al estudiar los problemas del conocimiento nos limitamos a enumerar las opiniones y puntos de vista de filósofos del pasado, oponiéndolas entre sí y comparándolas, en un ejercicio de erudición que puede resultar muy interesante para la historia de la filosofía, pero que es de escaso valor para la filosofía. (Me parece claro que la historia de la filosofía no es filosofía, al igual que la historia de la física no es ciencia física, aunque deba reconocerse que, en general y en diversas medidas, la noticia de la historia o desarrollo de una disciplina puede tener utilidad para la construcción actual de tal disciplina). Por otra parte, el enfoque meramente especulativo del conocimiento trata las cuestiones acerca del conocimiento con un vocabulario y un método que no tienen nada que ver con el conocimiento real que se da en los humanos. Una jerga filosófica, debidamente oscura y confusa, pretende ocultar el hecho de que las tesis ofrecidas no han sido sometidas a ninguna contrastación empírica ni tampoco comparadas con los datos científicos. Es posible así escribir gruesos libros sobre el conocimiento sin referencia alguna a la psicología o a la biología, ya que las doctrinas sustentadas son asunto meramente especulativo. Sirva como ejemplo el siguiente texto de Francisco Canals Vidal (1922-2009): «Porque si el entender es, en cuanto tal, acto que forma lo entendido dentro de sí mismo como expresado y dicho, en el medio intencional internamente enunciado en el que alcanza la realidad en intencionalidad expresa, ello es radicalmente posible por la íntima actualidad consistente en la autopresencia consciente o «subsistencia en sí» propia del ente espiritual» (Sobre la esencia del conocimiento, p. 694). 
Dentro del campo interdisciplinar de las ciencias cognitivas suele preferirse el término «cognición» al término «conocimiento», de tal manera que en vez de hablar de sujeto de conocimiento resulta preferible hablar de sujeto cognitivo. Ambos términos son en principio intercambiables, pero el uso actual (en conexión con las ciencias cognitivas) del término «cognición» implica dos novedades en la concepción del sujeto de conocimiento: 1) que los sujetos de conocimiento son procesadores de información, y 2) que los sujetos de conocimiento no se reducen a los seres humanos, tal como pensaba por ejemplo René Descartes (1596-1650), sino que incluyen diversos animales y diversas máquinas. Creo que debemos al genial lógico y matemático británico Alan Turing (1912-1954) la idea de que los sujetos de conocimiento son procesadores de información. En efecto, en su artículo «On Computable Numbers» (1937), nos habla de un computador (calculador) que observa símbolos, tiene estados mentales y escribe (o borra) símbolos. Este computador o calculador no es una máquina computadora (ya que los primeros computadores mecánicos, Colossus o ENIAC, son de 1943 y 1946 respectivamente) sino humanos, aunque Turing dice que «podemos construir una máquina para hacer el trabajo de este computador» (o. c. p. 137). Pero lo más importante es que este computador humano, al igual que la máquina computadora, reciben información, la transforman y pueden ofrecer nueva información. Por otra parte, el propio Turing, en «Computing Machinery and Intelligence» (1950) defendió la idea de que es posible que las máquinas computadoras tengan inteligencia, posibilidad que situó en el año 2000. Y en cuanto al conocimiento de animales distintos del hombre no parece haber duda entre los biólogos actuales, salvo determinar a partir de qué nivel biológico y qué tipo de conocimiento. Al respecto me parece básica la lectura del libro de Jean Piaget (1896-1980) Biología y Conocimiento (1969).

III. El Sujeto Cognitivo COMo SISTeMA Físico de símbolos (SFS).

El principio fundamental de las ciencias cognitivas es que la cognición es procesamiento de información o, dicho de otro modo, el concepto adecuado de sistema de procesamiento de información (SPI) es el supuesto básico común a las ciencias cognitivas. Y ya en torno a 1970 tanto psicólogos como informáticos advirtieron tal hecho, como, por ejemplo, Peter Lindsay y Donald Norman en su obra clásica Human Information Processing: An Introduction to Psychology (1972).

En todo caso los autores centrales al respecto son, por un lado, el informático y psicólogo Allen Newell (1927-1992) y, por otro lado, el informático y economista, que obtuvo el Premio Nobel de Economía en 1978, Herbert Simon (1916-2001). Los trabajos principales son los tres siguientes: 1) Human Problem Solving (1972) de Newell y Simon, donde se define un sistema de procesamiento de información (SPI), 2) «Computer Science as Empirical Inquiry» (1976) tam- 
bién de Newell y Simon, donde se define un sistema físico de símbolos (SFS), que es equivalente a un sistema de procesamiento de información (SPI), y 3) «Physical Symbol Systems» (1980) de Allen Newell (aunque Newell especifica que Simon podría haber figurado como coautor).

Para la noción de sistema físico de símbolos (SFS) podemos centrarnos en el último trabajo, que es la exposición más elaborada. Un SFS es una máquina que existe en un ambiente, donde hay objetos distribuidos en localizaciones. Tal SFS consta de una memoria, un conjunto de operadores, un control, una entrada y una salida. La conducta externa consta de las salidas que se producen en función de las entradas. Pero hay (además y sobre todo, cabría decir) una conducta interna que consta de la variación del estado interno a lo largo del tiempo, esto es, de la variación del estado de la memoria y del estado de control.

Podemos comentar esta caracterización de un sujeto cognitivo. En primer lugar, el término «máquina» se usa en sentido amplio, de modo que tanto un computador como un animal o un humano pueden ser entendidos como mecanismos. En segundo lugar, el sujeto cognitivo tiene entradas de información que son adecuadamente elaboradas mediante operaciones diversas (en el esquema de Newell figuran cinco operadores). En tercer lugar, hay una unidad de control en conexión con la memoria (información disponible acumulada), la cual actúa sobre los operadores de salida (Newell anota otros cinco operadores). Y en cuarto lugar, el SFS tiene órganos de recepción de información así como órganos motores.

También podemos hacer una ejemplificación concreta en términos de conocimiento humano. Los receptores son básicamente los cinco sentidos, es decir, ojos, oídos, nariz, paladar y piel. Los operadores son los medios de procesamiento de esa información que se realiza mediante el tálamo y mediante la corteza cerebral. El control quizás se realice en las zonas encefálicas frontales, en conexión con la memoria almacenada en las zonas de asociación temporales, parietales y frontales. A su vez, las operaciones de las salidas parecen estar iniciadas en la corteza frontal en conexión con los ganglios basales y desencadenadas por las cortezas motoras. Finalmente nuestros miembros, tronco y cabeza, o nuestra boca (lenguaje hablado) ejecutan las salidas motoras.

\section{COGNICIÓN EN SENTIDO AMPLIO Y COGNICIÓN PROPIA.}

Una vez establecido que los sujetos cognitivos pueden ser no sólo los humanos sino también máquinas computadoras así como otros seres vivos diferentes de los humanos, resulta necesario establecer si cualquier máquina tiene cognición y si cualquier ser vivo tiene cognición.

Respecto del segundo punto, el biólogo chileno Humberto Maturana ha sostenido, en «Biology of Cognition» (1980): «Los sistemas vivos son sistemas cognitivos, y vivir en cuanto proceso es un proceso de cognición. Tal enunciado 
es válido para todos los organismos, con y sin sistema nervioso» (o. c. p.13). Según ello cualquier ser vivo, perteneciente a cualquiera de los cinco reinos (bacterias, protistas, hongos, plantas y animales), tendría cognición.

A su vez, respecto del primer punto, el filósofo John Searle (crítico con la idea de que existan máquinas realmente inteligentes) cuenta que, en cierta ocasión, preguntó a John McCarthy (1927-2011), quien defendía que máquinas simples como los termostatos tienen creencias (y por ende cognición), qué creencias tenía su termostato, y McCarthy contestó: «Mi termostato tiene tres creencias. Mi termostato cree que hace aquí demasiado calor, que hace aquí demasiado frío, y que se está bien aquí» («Minds and Brains without Programs», p. 211).

En lo que se refiere a los seres vivos, cabría en efecto generalizar al máximo la noción de cognición y, por ejemplo, entender la fotosíntesis como un fenómenos de cognición en las plantas en cuanto recepción de información bajo forma de luz. Sin embargo, parece más razonable entender los fenómenos de cognición como ligados a la recepción, tratamiento y entrega de información. (Si se pide una noción de información, personalmente creo que podría ser suficiente anotar que información es cualquier cambio físico seleccionado por su eficacia o interés por su receptor).

En el caso de los seres vivos, los animales vertebrados (peces, anfibios, reptiles, aves y mamíferos) poseen órganos y funciones que posibilitan este intercambio activo de información, tal como se advierte en la posesión de un sistema nervioso central, con alto grado de cefalización y mielinización, aunque también debe anotarse que algunos animales invertebrados, como artrópodos y moluscos (por ejemplo, la famosa Aplysia) poseen ganglios neuronales de interés.

A su vez, en lo que se refiere a las máquinas, habitualmente y a lo largo de la historia se ha pensado que las máquinas no piensan y, por ende, no tienen cognición. Por ello el libro de 1979 de Pamela McCorduck titulado Machines Who Think constituyó un desafío y un hito definitivo en nuestra manera de pensar acerca de lo que piensa. Ciertamente esta obra se refería a las máquinas computadoras y, en especial, a los computadores inteligentes. Pero también en 1979 Hans Moravec, científico robótico austriaco-americano, publicó un artículo titulado «Today's Computers, Intelligent Machines and our Future», donde, que yo sepa, se defiende por primera vez la idea no sólo de la inteligencia de algunos robots sino incluso la posibilidad de que alcancen un conocimiento semejante al humano.

Pero conviene aclarar las cosas. Tal como he indicado en mi trabajo «Filosofía de la robótica inteligente» (p. 330), puede haber cierta confusión, y de hecho la hay, entre lo que es un simple mecanismo, una máquina que habitualmente no calificamos de robot, un robot no-inteligente y un robot inteligente. 
Podemos poner los ejemplos correspondientes. Un grifo es sin duda un simple mecanismo que precisa constantemente de un operador ajeno. Aspiradoras, neveras, lavadoras y lavaplatos son máquinas que no solemos llamar robots, aunque no me parece improcedente llamarlos así, ya que el término "robot» fue creado por el escritor bohemio Karel Chapek (1890-1938), en 1920, para referirse a máquinas que trabajan al servicio de los humanos. Sin embargo, esas máquinas señaladas, aunque no su adquisición masiva, existen antes de la divulgación de la palabra «robot». En cambio, las máquinas empleadas en las líneas de montaje de las fábricas de automóviles de USA o Japón, a partir de 1961, se llaman robots, aunque no son inteligentes, ya que se limitan a repetir mecánicamente una tarea fija. Y finalmente el robot Shakey, desarrollado desde 1966 en el Stanford Research Institute por Charles Rosen (1927-2002) es un ejemplo pionero de robot inteligente, ya que localizaba objetos, iba a buscarlos sorteando obstáculos y los empujaba hasta lugares determinados.

De lo expuesto se deduce que hay niveles diversos de cognición, entendida como manipulación de información. La información, por lo de pronto, puede ser recibida y devuelta al entorno con escaso procesamiento, o bien con menor o mayor elaboración en conexión con una unidad de control y su memoria. Por ejemplo, una sensación asfixiante de calor producirá normalmente abundante sudor, pero también puede producir nuestra acción de tomar una ducha fría para aliviar el calor.

Pero podemos hablar incluso de cognición propia y tal sería la cognición inteligente. Los lógicos tradicionales, como el portugués Juan de Santo Tomás (1589-1644), distinguían tres operaciones del intelecto o inteligencia: concebir, juzgar y razonar. En palabras actuales, la cognición propia o inteligente comprende la tipificación de la información (conceptos), la comparación de información (juicios o creencias) y, de manera especial, la obtención de nueva información a partir de otra ya disponible (inferencias). A su vez, las inferencias pueden ser de tres clases: 1) meramente inductivas o generalizadoras, 2) deductivas o demostrativas, y 3) abductivas o explicativas.

Y si comparamos máquinas computadoras con computadores humanos, actualmente encontramos que los programas de redes neuronales artificiales permiten a las máquinas generalizar tan bien como lo hacen los humanos, que los programas de demostración automática son frecuentemente superiores a los de los humanos normales, aunque las capacidades conjeturales y explicativas de los seres humanos siguen siendo superiores a las de las máquinas.

Desde el punto de vista funcional, la cognición en humanos, animales o máquinas también comprende grados. En primera instancia, la cognición permite una simple y pasiva adaptación al entorno, como ocurre típicamente en el mimetismo del camaleón. En segunda instancia, la cognición permite un aprendizaje en el dominio del entorno, tal como sucede con el robot Curiosi- 
ty, situado desde 2012 en Marte para la exploración del planeta. Y en tercera instancia, la cognición permite la creación de entornos tal como pasa con la construcción por los humanos de aviones y aeropuertos, buques y puertos, o automóviles y autopistas. Justamente el salto en la creación de entornos propio de los humanos indica claramente, a mi entender, el salto cognitivo que se produce desde nuestro primos primates (bonobos, chimpancés, orangutanes y gorilas) hacia nosotros mismos.

\section{REFERENCIAS BIBLIOGRÁFICAS}

BOBROW, DANIEL G., COLLINS, ALLAN (eds.), Representation and Understanding. Studies in Cognitive Science, Academic Press, Londres, 1975.

CANALS VIDAL, FRANCISCO, Sobre la esencia del conocimiento, Promociones Publicaciones Universitarias, Barcelona, 1987.

LINDSAY, PETER, NORMAN, DONALD, Human Information Processing: An Introduction to Psychology, Academic Press, Nueva York, 1972.

MCCORDUCK, PAMELA, Machines Who Think, Freeman, Nueva York, 1979.

MARTÍNEZ-FREIRE, PASCUAL F., «El impacto de las ciencias cognitivas en la filosofía del conocimiento», P. F. Martínez-Freire (coord.), Filosofía y ciencias cognitivas, Philosophica Malacitana, Suplemento no 3, 1995, pp. 51-66.

____ «Filosofía de la robótica inteligente», Antonio Diéguez y José María Atencia (coords.), Genes y Máquinas, Universidad de Málaga, Málaga, 2006, pp. 327-347.

____, La importancia del conocimiento. Filosofía y ciencias cognitivas ( $2^{\mathrm{a}} \mathrm{ed}$.), Netbiblo, La Coruña, 2007.

MATURANA, HUMBERTO, «Biology of Cognition», H. Maturana y Francisco Varela, Autopoiesis and Cognition, Reidel, Dordrecht, 1980, pp. 1-58.

MORAVEC, HANS, «Today's Computers, Intelligent Machines and our Future», Analog, 99, 2, febrero 1979, 59-84.

NEWELL, ALLEN, «Physical Symbol Systems», Cognitive Science, 4, 1980, 135-183.

NEWELL, ALLEN, Y SIMON, HERBERT, Human Problem Solving, Prentice-Hall, Englewood Cliffs (NJ), 1972.

___ «Computer Science as Empirical Inquiry: Symbols and Search», Communications of the Association for Computing Machinery, 19, marzo de 1976, 113-126.

NORMAN, DONALD A., RUMELHART, DAVID E. (eds.), Explorations in Cognition, Freeman, San Francisco, 1975.

PIAGET, JEAN, Biología y Conocimiento (trad. Fco. González Aramburu), Siglo XXI, Madrid, 1969.

SANTO TOMÁS, JUAN DE, Ars logica seu de forma et materia ratiocinandi, B. Reiser (ed.), Marietti, Casali, 1948. 
SEARLE, JOHN, «Minds and Brains without Programs», Colin Blakemore y Susan Greenfield (eds.), Mindwaves, Blackwell, Oxford, 1987, pp. 209-233.

TURING, ALAN M., «On Computable Numbers», Martin Davis (ed.), The Undecidable, Haven, Hewlett (New York), 1965, pp. 116-154. , «Maquinaria computadora e inteligencia» (trad. Fco. Martín), Alan Ross Anderson (ed.), Controversia sobre mentes y máquinas, Orbis, Barcelona, 1987, pp. 11-52.

Pascual F. Martinez-Freire es catedrático emérito de Lógica y Filosofía de la Ciencia en la Universidad de Málaga.

Lineas de investigación:

Filosofía de las Ciencias Cognitivas, Filosofía de la Mente, Neurofilosofía.

Publicaciones recientes:

«Representación y creación mental», Revista Mexicana de Investigación en Psicología, 9, 2, 2017, 139-146.

«Valores científicos y democracia ética», Ludus Vitalis, XXIV, 46, 2016, 171-174.

«Cerebro: funcionalidad, dinamismo e intencionalidad», Studia Poliana, 18, 2016, 13-26.

Correo electrónico: freire@uma.es 
